\title{
Melanoma incidence trends in Limburg after screening and prevention campaigns
}

\author{
by \\ Vanstraelen $\mathrm{D}^{1 \dagger}$, Deleu $\mathrm{H}^{2}$, Van Robays $\mathrm{J}^{2}$, Dhollander $\mathrm{D}^{3,4}$, Cloes $\mathrm{E}^{3,5}$, Lousberg $\mathrm{D}^{3,6}$, \\ Kuipers $\mathrm{G}^{7}$, Adriaens $\mathrm{B}^{7}$, Mebis $\mathrm{J}^{1}$, Mathei $\mathrm{C}^{3,6}$, Buntinx $\mathrm{F}^{3,6}$
}

\begin{abstract}
Background

Malignant melanoma is a skin cancer of which the incidence is said to be raising in recent years. Prevention may result in decrease of the incidence. Early detection may result in the detection of earlier stages of the disease. In Limburg a combined campaign of prevention and screening has been performed in recent years and its effects should be evaluated.
\end{abstract}

\section{Objectives}

To study time trends of malignant melanoma incidence and the evolution of stages of detected melanomas and to relate them to interventions in previous years.

\section{Methods}

An ongoing multi-faceted intervention, including information as well as screening invitations (Euromelanoma project) was implemented in 1999. Time trends of malignant melanoma were studied on the basis of data from the Limburg Cancer Registry (LIKAR) of 1996-2005. Stages were studied on the basis of an additional clinical registration completed by pathologists and dermatologists.

\section{Results}

Over the years 1996-2005, the crude incidence rate of malignant melanoma was $6.8 / 100,000$ patient-years in males and 11.6 in females, with a European standardized incidence rate (ESR) of 6.4 in males and 10.5 in females. The incidence progressively increased with age.

\footnotetext{
$\bar{\dagger}$ Dr D. Vanstraelen has passed away after submission of this manuscript, but before it could be published.

1 Limburg Oncological Centre, Virga Jesse Ziekenhuis, Hasselt, Belgium

2 Ziekenhuis Oost Limburg, Genk, Belgium

${ }^{3}$ Limburgse Kankersamenwerking (LIKAS), Hasselt, Belgium

${ }^{4}$ Christelijke Mutualiteit Hasselt

5 Expertisecentrum Digitale Media (EDM), University of Hasselt, Hasselt, Belgium

6 Catholic University of Leuven, Department of General Practice, Clinical Epidemiology Unit, Leuven, Belgium

7 Province of Limburg, Department of Health, Hasselt, Belgium

Correspondence: frank.buntinx@med.kuleuven.be
} 
In males, the ESR initially decreased, followed by a significant increase from 3.0 in 1998 to 7.6 in 2005. On average this is a yearly increase of 0.5 per 100,000 persons $(p=0.04)$. In females the curve fluctuates, resulting in almost similar rates in 1996 and 2004 ( $p=0.85)$, but with a sudden increase from 9.2 to 15.8 between 2004 and 2005.

Neither the Breslow nor the Clarck classification showed any significant change over the registration period. The proportion of lymph node invasion, metastasis and ulcerations did not change.

\section{Discussion}

With the exception of a small and clinically irrelevant increase in ESR in males, no changes over time were detected in incidence or stage distribution. The effect of the intervention seems limited.

\section{Keywords}

Melanoma, screening, incidence trends

\section{Introduction}

Malignant melanoma is a relatively rare malignancy of the skin that seems to become more frequent over the years (1). The main cause is considered to be skin burn in sensitive people, especially in children (2), although most cancers will be detected above age 25 in females and age 35 in males (3). In far too many cases, melanomas are diagnosed in advanced stages and prognosis has been bad although it recently improved after the introduction of resection policies including a very broad zone of normal tissue.

Starting in 1999, the provincial health authorities of the Belgian province of Limburg, together with regional health care workers and the funding organization TOKAP, adopted a comprehensive approach towards the population in order to prevent and detect melanomas early. The approach included sun protection information campaigns aimed at the population at large as well as at elective target groups, and yearly invitations for screening, within the framework of the Euromelanoma day.

Information campaigns can only be expected to show results after decennia, unless the campaign also includes guidelines for self-examination of the skin and screening by physicians, which is the case here.

Yearly screening of the total population, including self-examination of the skin can be expected to result in a higher incidence during a period of two years (first round effect), followed by a gradual decrease towards the original level. As a total population screening is unrealistic, the initial increase will be spread over a much longer period, maybe ten years or more and the incidence peak will be much lower and therefore difficult to detect. Even in this case, however, it can be expected that melanomas will be detected earlier, resulting in the detection of tumours of an earlier stage. 
After ten years of campaigning, we used data of the Limburg Cancer Registry (LIKAR) (3) as well as a more detailed additional registration of melanomas to evaluate the time trend of both the incidence and the distribution of the stages over the years and tried to relate this to the screening and prevention campaigns.

\section{Methods}

\section{Design}

We used a time trend analysis, comparing yearly incidence figures and tumour stages of melanomas diagnosed between 1996 and 2005.

\section{Population}

Our data relate to the population of the Belgian province of Limburg, situated in the northeast of Belgium. The territory covers $2422 \mathrm{~km}^{2}$ or $7.9 \%$ of the Belgian territory. On 1 January 2001 the population totalled 791,178, of which 395,579 men and 395,599 women. The degree of urbanisation is restricted: of the two largest cities Hasselt ( $n=68,058$ on $01 / 01 / 2001$ ) and Genk ( $n=62,842$ on $01 / 01 / 2001)$ constitute $17 \%$ of the total population. Both are situated in the area of Central Limburg. Forty-four percent of the population lives in 32 municipalities with 20,000 inhabitants or less, and $39 \%$ in 10 municipalities with 20,000 to 40,000 inhabitants (Figure1).

Figure 1: The distribution of the population
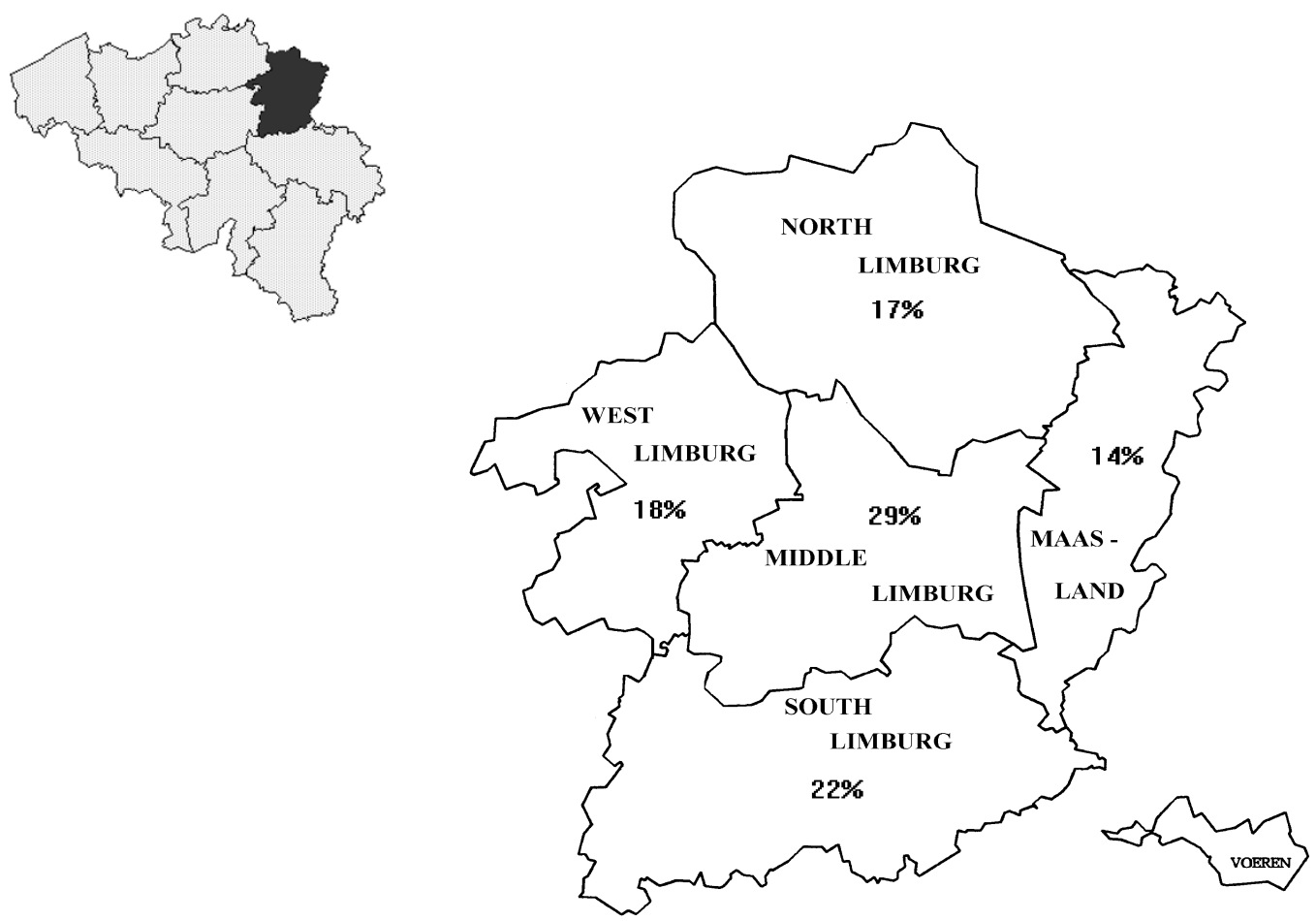


\section{Interventions}

The intervention was designed and operationalised by a multidisciplinary group including physicians, funders, and policy makers. It was progressively built up over the last ten years, and included a number of methods (to be divided into patient information/primary prevention) and screening (and target groups):

- The campaign called 'Be sensible in the sun' concentrated on taking appropriate precautions to prevent the emergence of malignant melanomas. It included the production and distribution of leaflets, brochures, a sundial (recently also available on the website), posters, postcards, sunscreen lotion labels, a sunscreen to be placed behind the windscreens of cars and lectures in different municipalities targeted at the general population.

- Additional posters, postcards, leaflets, and a competition were addressed at camp leaders, youth clubs, after-school childcare programmes, playground activities, sports clubs and event visitors. A question and answer game was used for class five primary school children as a waiting room activity during the school medical check-up.

- An inspirational folder was sent to local authorities and a life-size sun dial was made available to stimulate them to add their own activities to the common action of the province.

- Adapted promotional materials were distributed through well-baby clinics.

- A training course and a CD-ROM were developed for GPs.

- Similar to elsewhere in Europe, from 1999 onwards, in Limburg the 'Melanoma Monday' was organised yearly in the first half of May, as a method to encourage the population and especially sensitive people to present themselves for a skin examination by a dermatologist free of charge (4). More than half of the Belgian dermatologists collaborate on the initiative. The yearly number of participating patients is between 4000 and 5000, without any specific trend. The number and percentage of positive cases have descended from $25(0.9 \%)$ in 1999 to $8(0.2 \%)$ in 2003.

\section{Data collection}

Incidence rates were calculated from the LIKAR registry, which at the time of writing covers the years 1996-2005. The registry includes all cancers in inhabitants of the province of Limburg, which have been histologically or cytologically confirmed. A detailed description of its methods and procedures has been published elsewhere (3). More detailed information on each tumour was directly recorded from the pathologist and the referring clinician, in general the dermatologist. Until the year 2001, cases were retrieved from LIKAR, and both the pathologist and the clinician were asked to provide the additional information; from 2002 until 2005 data were prospectively identified by the pathologist. As a result these data are available until the year 2005. 
For each diagnosis of a malignant melanoma, the pathologist completed a form recording APO thickness, presence of tumour cells at the section margins, presence of ulceration, presence of micro metastasis and pathological TNM (pTNM) classification. The form was sent to the clinician, who was asked to add the location of the primary tumour, the Clark and Breslow stage, the AJCC and TNM classification and the presence of any known metastasis (5). All the involved physicians were informed about the goals of the project and the procedure to be followed via the loco-regional quality evaluation groups (LOK). Each Belgian clinician has to be a member of one of these groups in order to keep their certification. The anonymity of each physician's data towards the group was guaranteed.

The whole project was validated by the ethical review board of the Belgian work against cancer.

\section{Outcome measures}

The TNM classification of malignant melanoma is presented in table 1, and incorporates three main prognostic features: tumour thickness (Breslow), anatomical Clark levels and the absence or presence of ulceration (5).

Table 1: TNM classification of malignant melanoma

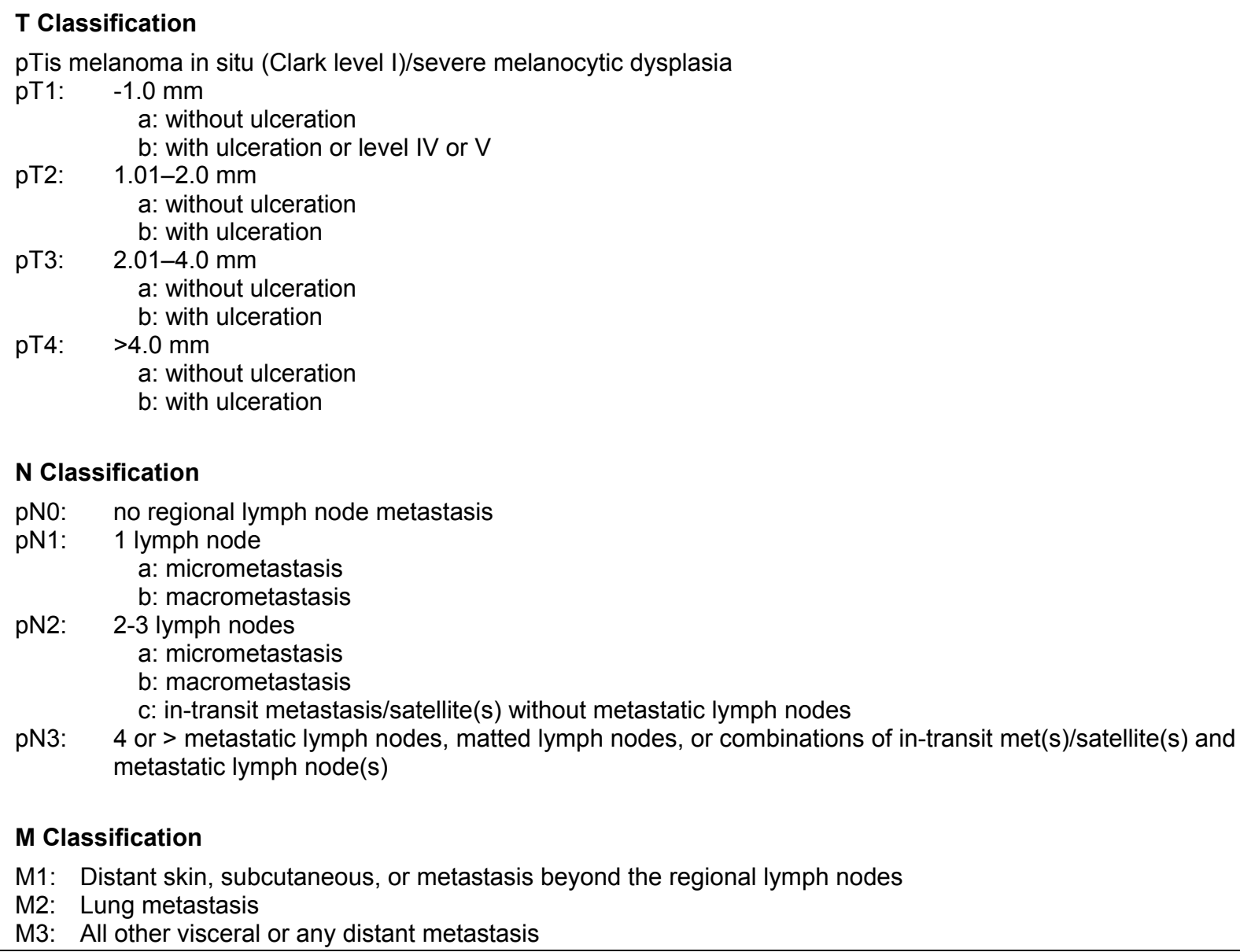

\section{Classification}

M1: Distant skin, subcutaneous, or metastasis beyond the regional lymph nodes

M2: Lung metastasis

M3: All other visceral or any distant metastasis 
The Breslow index is the tumour maximum vertical diameter from the top of the granular layer or ulcerated tumour surface to the deepest point of invasion. It is measured in millimeters.

The Anatomical Clark level is categorised from I to $V$ and subsequently includes the epithelium only (I), the papillary dermis (II), the papillary-reticular interface (III), the reticular dermis (IV), and the subcutaneous fat (V).

Ulceration (particularly if $>3 \mathrm{~mm}$ ) is an independent adverse prognostic factor, with a ten-year survival of around $50 \%$ in case of ulceration versus $78 \%$ if non-ulcerated.

Presence of tumour cells at the section margin or ulceration as seen during histological examination and known metastasis were recorded as either present or absent.

Analysis

For comparison, melanoma incidence rates have been stratified by gender and standardised for age according to the European standard population (ESR per 100,000 person-years). ESR values for males and females separately were linked to the year by simple linear regression analysis.

Tumour thickness and classifications were linked to the year by Kruskall-Wallis analysis (categorical dependent variable) or linear regression analysis (continuous dependent variable) and by chi ${ }^{2}$ testing or Kruskall-Wallis after categorisation of the years into three classes (1996-1999, 2000-2002, 2003-2005) (Table 2).

Table 2: Depth and stage of the tumours according to three time periods

\begin{tabular}{|c|c|c|c|c|c|}
\hline Classification & class & 1996-1999 & 2000-2002 & 2003-2005 & tota \\
\hline \multirow[t]{3}{*}{ Breslow $(\mathrm{mm})$} & $<0.5$ & 48 & 59 & 41 & 148 \\
\hline & $0.5-3$ & 114 & 90 & 84 & 288 \\
\hline & $>3$ & 21 & 30 & 21 & 72 \\
\hline \multirow[t]{5}{*}{ Clarck } & 1 & 12 & 19 & 11 & 42 \\
\hline & 2 & 36 & 41 & 36 & 113 \\
\hline & 3 & 67 & 42 & 54 & 163 \\
\hline & 4 & 48 & 58 & 38 & 144 \\
\hline & 5 & 11 & 11 & 9 & 31 \\
\hline \multirow[t]{4}{*}{$\mathrm{pN}$} & PNO & 158 & 145 & 165 & 468 \\
\hline & PN1 & 4 & 3 & 8 & 15 \\
\hline & PN2 & 1 & 0 & 0 & 1 \\
\hline & PN3 & 0 & 2 & 1 & 3 \\
\hline \multirow[t]{2}{*}{$\mathrm{pM}$} & PM0 & & & & \\
\hline & PM1 & 14 & 4 & 7 & 25 \\
\hline
\end{tabular}




\section{Results}

\section{Incidence rates}

Over a period of ten years, LIKAR recorded 735 malignant melanomas, 271 in males and 464 in females. This results in an overall crude incidence rate of $6.8 / 100,000$ patient-years in males and 11.6 in females or an ESR of 6.4 in males and 10.5 in females. The incidence progressively increases with age (Figure 2).

Figure 2: Incidence of malignant melanoma according to age and sex (LIKAR 1996-2005)

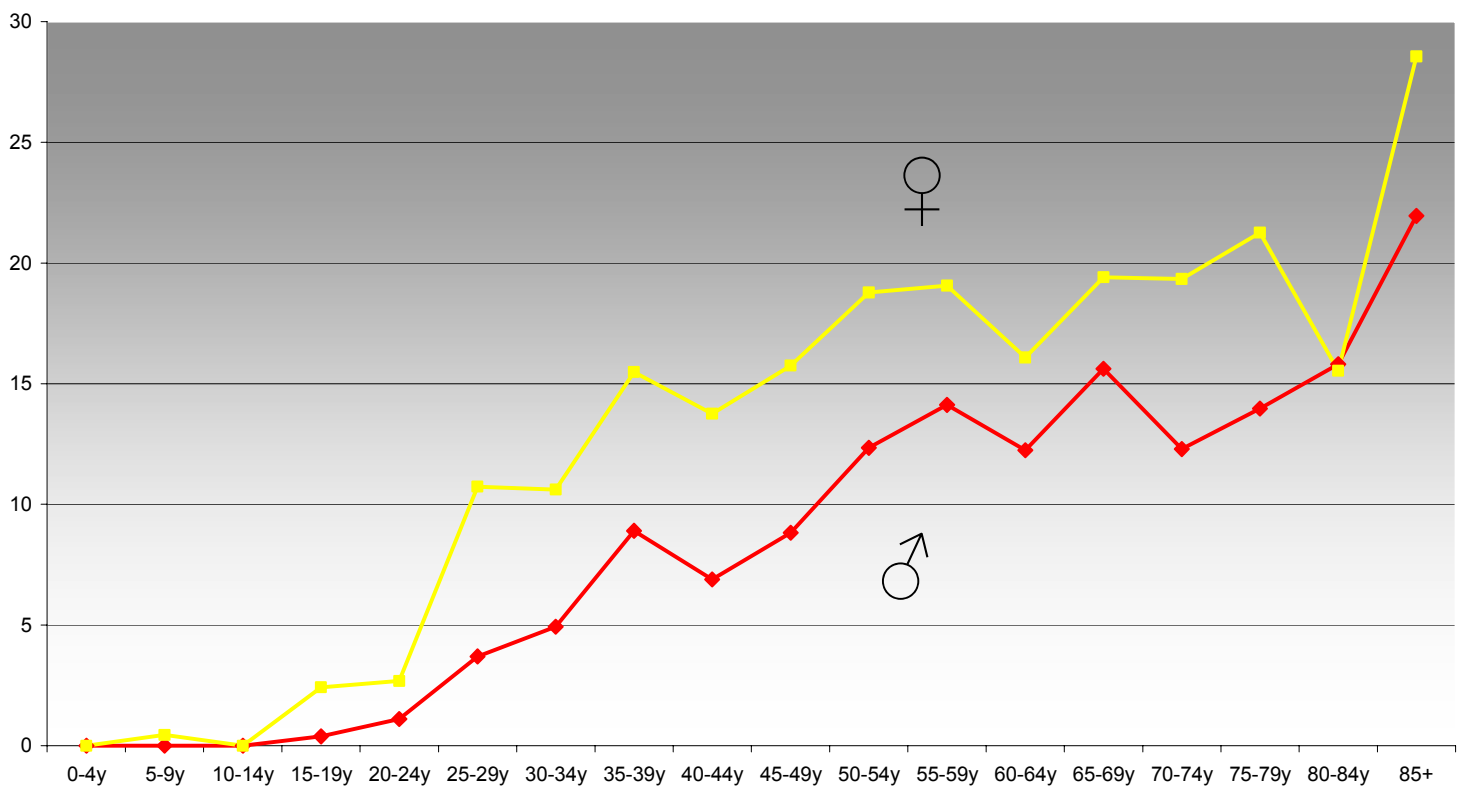

Figure 3: Time trend of the incidence (ESR/100,000 patient-years) of malignant melanoma in inhabitants of the Belgian province of Limburg (LIKAR 1996-2005)

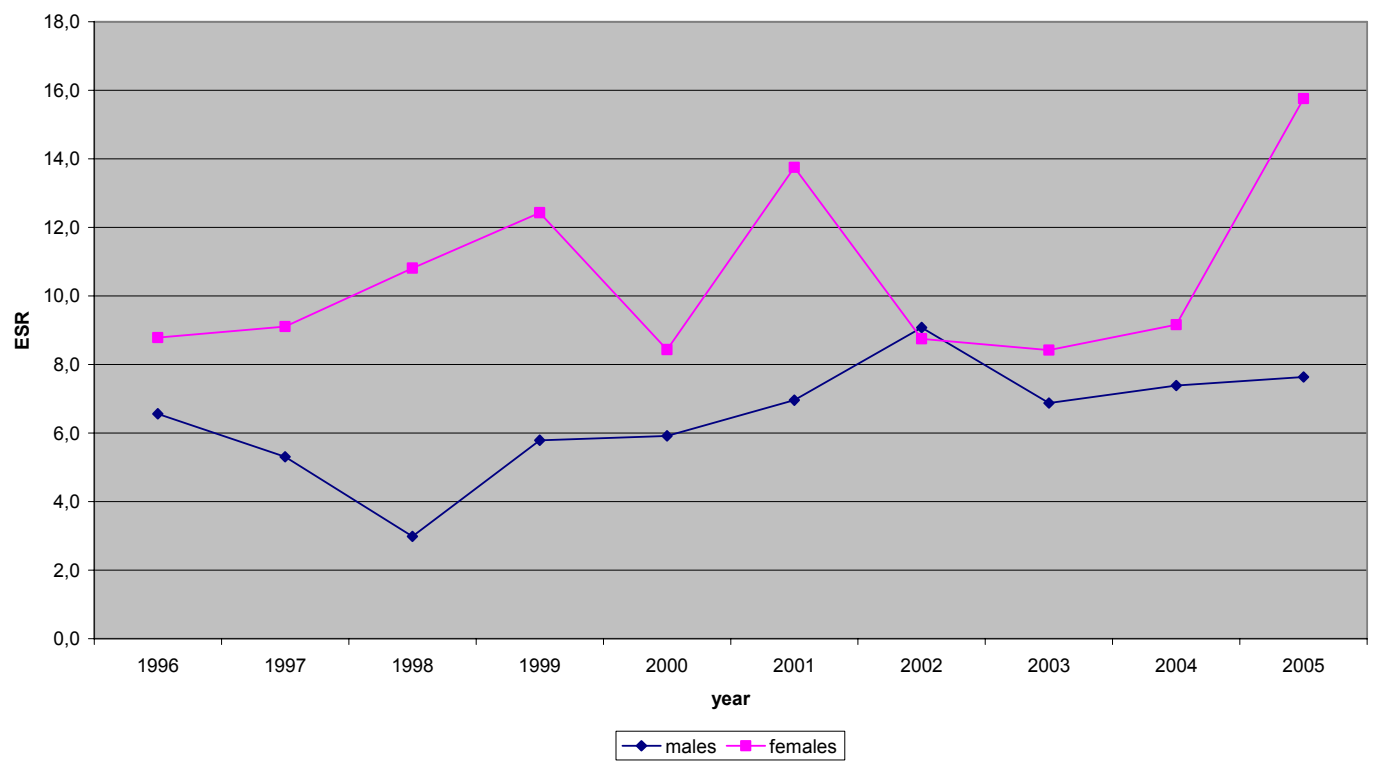


In males, the ESR initially decreased, followed by a steady and statistically significant increase from 3.0 in 1998 to 7.6 in 2005. On average this is a yearly increase with 0.5 per 100,000 persons $(p=0.04)$. The proportion of the variance in incidence explained by time is $55 \%$. In females the curve fluctuates, resulting in almost similar rates in 1996 and 2004 $(p=0.85)$, but with a sudden increase from 9.2 to 15.8 between 2004 and 2005 (Figure 3).

\section{Clinical registration}

Some detailed information was available for 601 patients. At least one measure of staging was available for 509 patients. There were 219 males and 362 females (gender missing in 20 cases). Their mean age was 55 years (range 18-92). Mean age and sex ratio were stable over the three time periods that were analysed.

There were 339 patients (56\%) with a reported diagnosis of superficial spreading of malignant melanoma, $96(16 \%)$ with a nodular melanoma, $51(9 \%)$ with a lentiginous melanoma, and $43(7 \%)$ with an in situ malignant melanoma. All other types counted less than $3 \%$ each. There was no increasing or decreasing trend in the relative occurrence of the most frequent types over the three time periods.

\section{Staging}

Depth of the lesion according to Breslow was available for 509 patients. Overall the depth was less than $0.5 \mathrm{~mm}$, between 0.5 and 3mm and more than 3mm in 149 (29\%), 293 (57\%) and $73(14 \%)$ cases, respectively. There was no relation between the depth and year of diagnosis $(p=0.40)$, nor between depth and the three time periods $\left(\mathrm{chi}^{2} \mathrm{p}=0.23\right)$ (Figure 4).

Figure 4: Depth of the lesion according to Breslow over the years

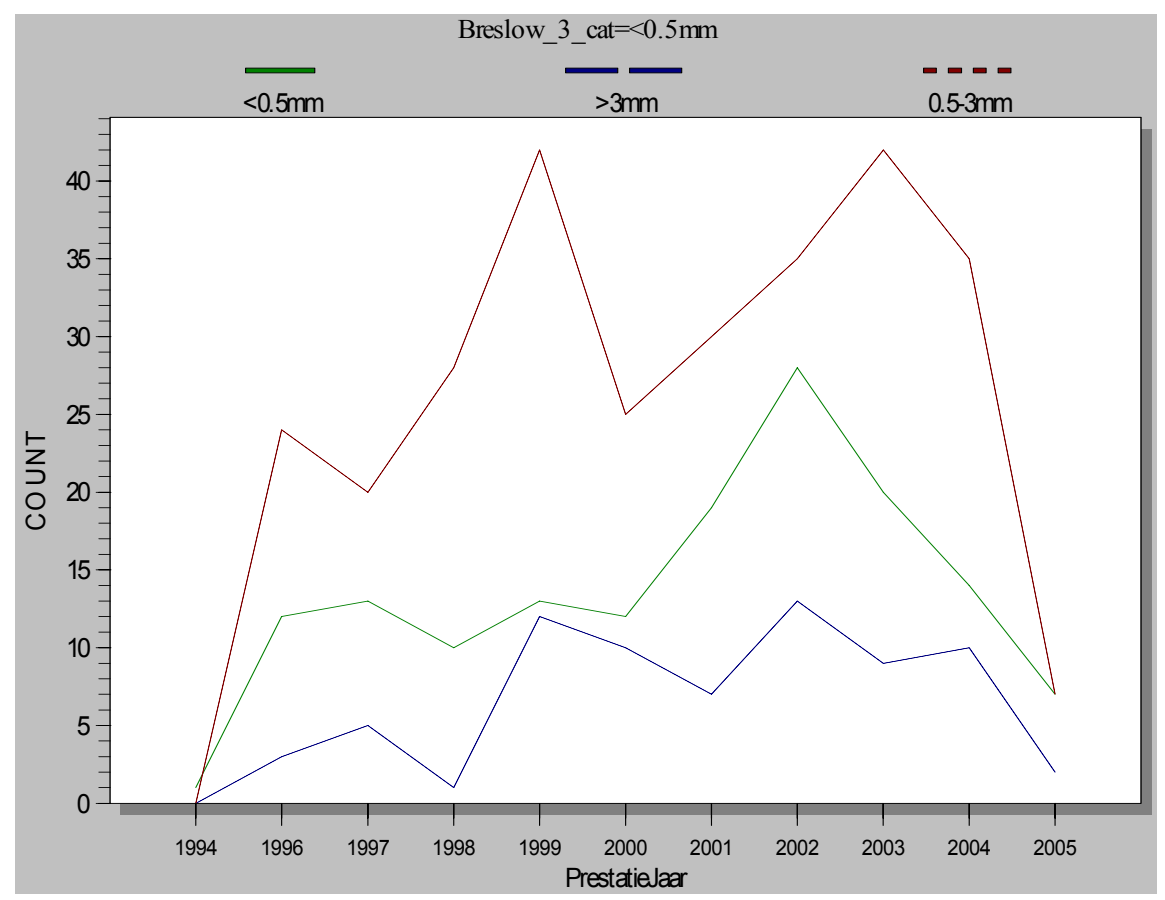


The classification according to Clark was available for 493 patients. Over the years the relative frequency of the different Clark classes fluctuated heavily. There was no increasing or decreasing trend (regression, $p=0.11$ ) (Figure 5). Neither was there a relation between the proportion of Clark classes and the time periods $\left(\mathrm{chi}^{2} \mathrm{p}=0.23\right)$.

Figure 5: Clark classes according to year of diagnosis

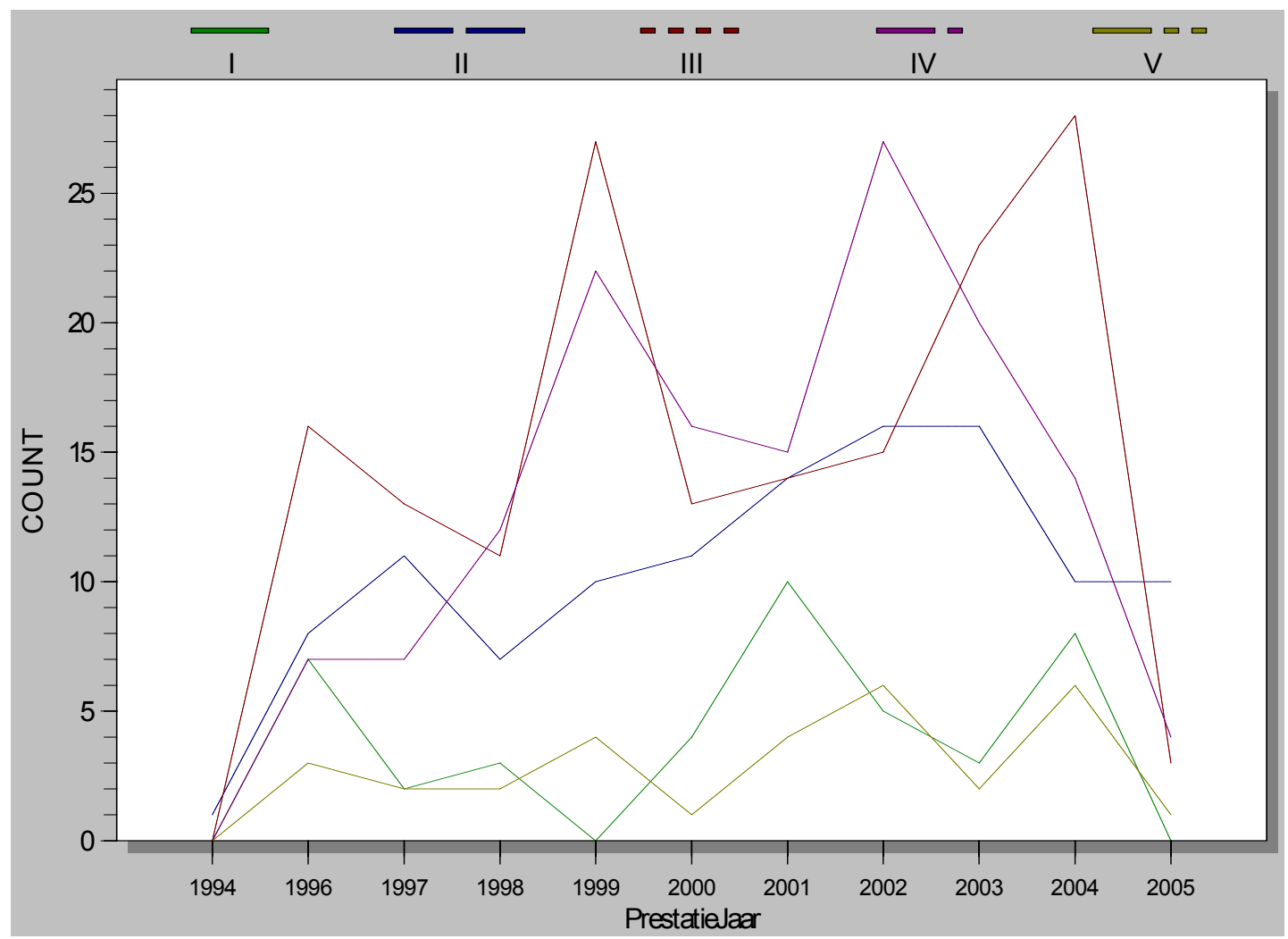

Lymph node invasion was registered in 163 cases and present in $19(12 \%)$. There was no time trend (ANOVA, $\mathrm{p}=0.23$ ). Risk of lymph node invasion was not related to gender (chi $p=0.48)$ or age (ANOVA, $p=0.87$ )

Clinically known metastasis was present at the time of diagnosis in $25(5 \%)$ cases. There was no relation between the proportion of metastases and gender $\left(\mathrm{chi}^{2} \mathrm{p}=0.69\right)$ or the year of diagnosis (ANOVA, $p=0.19$ ) (Figure 6). Risk of metastasis was significantly related, however, to increasing age (linear regression $p=0.003$ ), although the effect was limited. The proportion of metastases explained by age $\left(R^{2}\right)$ was only $2 \%$.

Ulceration was registered in 388 cases and present in $80(21 \%)$. There was no relation with gender $\left(\mathrm{chi}^{2} \mathrm{p}=0.68\right.$ ) or year of diagnosis (ANOVA $\mathrm{p}=0.86$ ). Risk of ulceration increased slightly, however, with increasing age $\left(p=0.002, R^{2}=2 \%\right)$. 
Figure 6: proportion of patients with a metastasis at the time of diagnosis

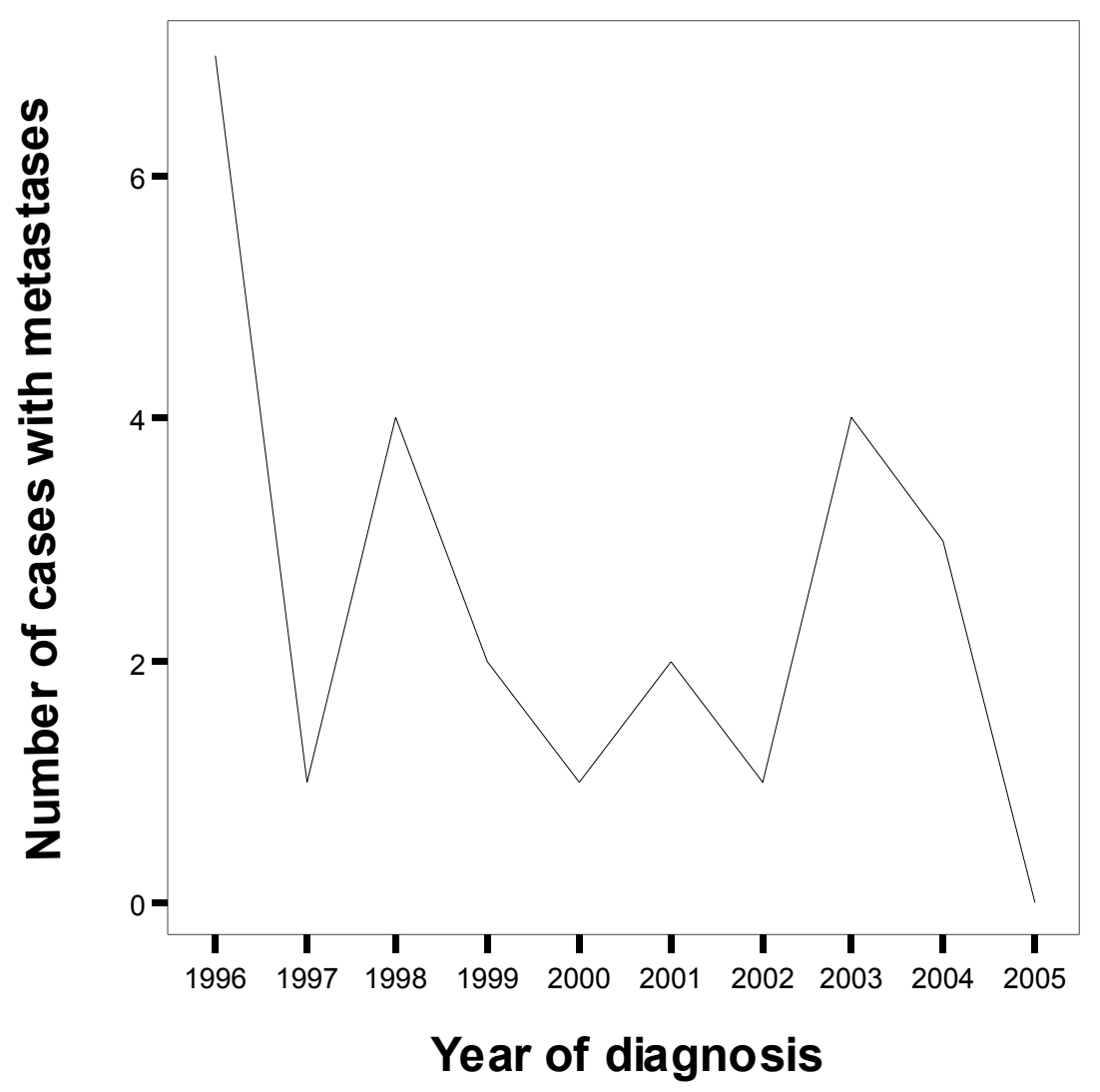

\section{Discussion}

The LIKAR ESRs for melanoma are lower compared to the recently published national data of 2005 (10.4 and 15.3 for males and females) (6). This may be caused by differences in registration procedures (LIKAR only includes tumours which are histologically or cytologically confirmed) or from a more rural population.

With ESR values between 5 and 15 per 100,000 people and per year, malignant melanoma is in both males and females a reasonably rare tumour. Nevertheless, it strikes about 54,000 people in Europe $(1,2)$ every year, and 37,000 patients around the world die from it annually.

This analysis was not intended and not able to measure the effect of the sun protection information part of our intervention. This can only be evaluated on a long term basis. However, we expected yearly screening of (part of) the population and encouraging people to perform self-examination of the skin to result in a temporary higher incidence of melanoma (first round effect), followed by a gradual decrease towards the original level. As a total population screening is unrealistic, this increase may be spread over a period of many years and the incidence peak may be low and therefore difficult to detect. Even in this case, however, we expected that melanomas would be detected earlier, resulting in a gradual evolution towards detecting tumours at an earlier stage. 
In females we could not identify any significant time trend over the ten years of registration. This may be a result of either absence of such relation or of low numbers. However, combining the cases of three groups of three or four years also gives the same result, while this should result in sufficient power to detect any clinically relevant change. The sudden increase in the year 2005 is considered to be coincidental and for the time being, neglected. In males, the ESR initially decreased, followed by a steady and statistically significant yearly increase of 0.46 per 100,000 persons (Figure 3). This increase means more than a doubling of the incidence over seven years, but absolute numbers are low and clinically this remains almost negligible. Over the whole of the ten years the trend even fades. A similar pattern has been identified in the registry of the Integrale kankerstichting Limburg (IKL) and Zuid (IKZ), our neighbours from just across the Dutch border (7). The increase may be the result from all information efforts, especially those encouraging people to have their skin examined on a regular basis. Similar results have been observed elsewhere. Janda et al. found significantly increasing screening rates, especially in men over 50, after a screening intervention in Australia. Those men, who were more likely to be screened, had skin lesions removed previously or had identified personal risk factors for melanoma (8). We do not know if a similar pattern shows in our population. Moreover, in our view, this does not sufficiently explain the between-gender difference in trend.

In spite of the yearly collaboration of more than half of the dermatologists, the impact of the Euromelanoma day seems very limited. This is understandable in view of the small number of tumours detected (4).

Regarding the pathology distribution, most of the malignant melanomas were superficially spread (56\%), $16 \%$ percent presented with nodular melanoma. This distribution is comparable with the literature (9).

For the 509 patients whose Breslow index was noted, $29 \%$ percent had a Breslow index $<0.5$ $\mathrm{mm}, 57 \%$ between 0.5 and $3 \mathrm{~mm}$. It is obvious that the prognosis is better with less thickness. Patients with tumours less than $0.76 \mathrm{~mm}$ thickness have a likelihood of recurrence of less than $1 \%$ in 10 year (10). We did not find a trend towards lesser Breslow thickness in our study, nor a lower Clark index after the screening campaign. So no trend towards a better prognosis of melanoma could be identified by our information and screening campaign. Here also, insufficient statistical power may be a problem for the initial analysis, but regrouping to three periods of three or for years did not change the picture.

Because melanomas are rare tumours and physicians had to register a limited number of cases each, it was expected that they would be motivated to do so. We elaborated a user friendly registration map and introduced the melanoma registration project in the LOK, where physicians come together per discipline to discuss professional matters. It proved extremely difficult, however, to motivate the dermatologists to register. This part of the initial planning was therefore abandoned. On the contrary the technique of extracting the melanomas from the LIKAR-register, a working cancer register in the province, followed by enriching the data for each melanoma by interrogating the pathologists on the case was a much better ap- 
proach. It is important to collect the right and relevant data on each melanoma. So our registration form had to be complete, appealing and not too large or difficult to fill in. We therefore did not ask for the number of mitoses in the tissue sample, an item we probably will include in the dataset in the future.

Our study was intended to be a first step to an ongoing tumour-specific register for melanomas that would enable us to follow the evolution of the melanomas in our province. Not only the incidence, but also the characteristics of the tumours can change. Those changes could be a result of changing sun-exposure habits or a change in screening efforts. A specific indepth registration of characteristics of melanomas is the only possibility to give us that information.

This study identified a small scale effect of the intervention on the melanoma incidence in males, but not in females and without any effect on the distribution of the tumour stages. This intervention was targeted at the population as a whole. It has been suggested that effectiveness of skin cancer screening may be higher if targeted at high-risk persons only. We therefore consider targeting our actions in the future at people who are at increased risk, either by their personal skin characteristics or by occupation. This especially includes people frequently working in open air. Selection of such high risk people can easily be done by occupational medicine and by general practitioners. As dermatologists may be better qualified to identify melanoma and atypical naevi compared to non-dermatologists (11), the use of dermatoscopy by non-specialised clinicians may improve their yield, at least in selecting patients with an increased risk of abnormality from the large bunch of patients with a clearly normal picture (12). Pre-selected patients may then be more efficiently examined by dermatologists.

\section{Acknowledgements}

The Limburg Cancer Registry (LIKAR) has been funded by the Flemish Government. Both LIKAR and the Limburg melanoma project were supported by the Limburg provincial authorities. We thank all pathologists and dermatologists for their work, which made this analysis possible.

\section{Literature}

1. De Vries E, Coebergh JW. Cutaneous malignant melanoma in Europe. Eur J Cancer 2004; 40(16):235566

2. Osterlind A. Epidemiology on malignant melanoma in Europe. Acta Oncol 1992; 31(8):903-8

3. Lousbergh D, Cloes E, Op de Beeck L, Rummens JL, Vanden Brande J, Faes C, Bruckers L, Molenberghs G, Dhollander D, Kellen E, Hensen K, Lathouwers D, Meekers E, Buntinx F. Incidence of cancer in the Belgian Province of Limburg 1996-2005. Hasselt, LIKAS:2007

4. http://www.euromelanoma.org

5. Allan DC. Histopathology reporting. Guidelines for surgical cancer. London, Springer: 2000

6. http://www.kankerregister.be/

7. http://www.ikcnet.nl/page.php?id=160; accessed on 10/11/2007 
8. Janda M, Youl PH, Baade PD, Elwood M, Ring IT, Aitken JF. What motivates men age $>$ or $=50$ years to participate in a screening program for melanoma? Cancer 2006;107: 815-23

9. Austoker J. Cancer prevention in primary care: Melanoma: prevention and early diagnosis. BMJ 1994; 308: 1682-6

10. Blois MS, Sagebriel RW, Abarbanel RM, et al. Malignant melanoma of the skin: I. The association of tumour depth and type, and patient sex, age and site with survival. Cancer 1983; 52: 1330-41

11. Ferrini R, Perlman M, Hill L: Screening for skin cancer. American College of preventive medicine practice policy statement, Am C Prev Med 1998; 14: 80-2

12. Westerhoff $\mathrm{K}, \mathrm{McC}$ arthy $\mathrm{WH}$, Menzies SW. Increase in the sensitivity for melanoma diagnosis by primary care physicians using skin surface microscopy. Br J Dermatol 2000;143:1016-20 